\title{
Pathogenicity, Transmission and Antigenic Variation of H5N1 Highly Pathogenic Avian Influenza Viruses
}

\author{
Peirong Jiao 1, 2,3,4t, Hui Song 1, 2,3,4t, Xiaoke Liu 1, 5 , Yafen Song 1, 2,3,4, Jin Cui 2, 3, 4, \\ Siyu Wu 1, 2,3,4, Jiaqi Ye ${ }^{1,2,3,4}$, Nanan Qu 1, 2,3,4, Tiemin Zhang ${ }^{6}$ and Ming Liao ${ }^{1,2,3,4 *}$ \\ ${ }^{1}$ National and Regional Joint Engineering Laboratory for Medicament of Zoonosis Prevention and Control, Guangzhou, \\ China, ${ }^{2}$ Key Laboratory of Animal Vaccine Development, Ministry of Agriculture, Guangzhou, China, ${ }^{3}$ Key Laboratory of \\ Zoonosis Prevention and Control of Guangdong, Guangzhou, China, ${ }^{4}$ College of Veterinary Medicine, South China \\ Agricultural University, Guangzhou, China, ${ }^{5}$ Pulike Biological Engineering Inc., Luoyang, China, ${ }^{6}$ College of Engineering, \\ South China Agricultural University, Guangzhou, China
}

OPEN ACCESS

Edited by:

Akio Adachi,

Tokushima University Graduate School, Japan

Reviewed by:

Huaguang Lu,

The Pennsylvania State University,

USA

Eri Nobusawa,

National Institute of Infectious

Diseases, Japan

*Correspondence:

Ming Liao

mliao@scau.edu.cn

${ }^{\dagger}$ These authors have contributed equally to this work.

Specialty section:

This article was submitted to

Virology,

a section of the journal

Frontiers in Microbiology

Received: 09 November 2015

Accepted: 18 April 2016

Published: 06 May 2016

Citation:

Jiao $P$, Song $H$, Liu X, Song Y, Cui J,

Wu S, Ye J, Qu N, Zhang T and Liao M

(2016) Pathogenicity, Transmission

and Antigenic Variation of $\mathrm{H} 5 \mathrm{~N} 1$

Highly Pathogenic Avian Influenza

Viruses. Front. Microbiol. 7:635.

doi: 10.3389/fmicb.2016.00635
H5N1 highly pathogenic avian influenza (HPAl) was one of the most important avian diseases in poultry production of China, especially in Guangdong province. In recent years, new H5N1 highly pathogenic avian influenza viruses (HPAIV) still emerged constantly, although all poultry in China were immunized with H5N1 vaccinations compulsorily. To better understand the pathogenicity and transmission of dominant clades of the H5N1 HPAIVs in chicken from Guangdong in 2012, we chose a clade 7.2 avian influenza virus named A/Chicken/China/G2/2012(H5N1) (G2) and a clade 2.3.2.1 avian influenza virus named A/Duck/China/G3/2012(H5N1) (G3) in our study. Our results showed that the chickens inoculated with $10^{3} \mathrm{EID}_{50}$ of G2 or G3 viruses all died, and the titers of virus replication detected in several visceral organs were high but different. In the naive contact groups, virus shedding was not detected in G2 group and all chickens survived, but virus shedding was detected in G3 group and all chickens died. These results showed that the two clades of H5N1 HPAIVs had high pathogenicity in chickens and the contact transmission of them was different in chickens. The results of cross reactive $\mathrm{HI}$ assay showed that antigens of $\mathrm{G} 2$ and $\mathrm{G} 3$ were very different from those of current commercial vaccines isolates (Re-4, Re-6, and D7). And to evaluate the protective efficacy of three vaccines against most isolates form Guangdong belonging to clade 2.3.2. 1 in 2012, G3 was chosen to challenge the three vaccines such as Re-4, Re-6, and D7. First, chickens were immunized with $0.3 \mathrm{ml} \mathrm{Re}-4$, Re-6, and D7 inactivated vaccines by intramuscular injection, respectively, and then challenged with $10^{6} \mathrm{EID}_{50}$ of G3 on day 28 post-vaccination. The D7 vaccine had 100\% protection against G3 for chickens, the Re-6 vaccine had $88.9 \%$, and the Re-4 vaccine only had $66.7 \%$. Our results suggested that the D7 vaccine could prevent and control H5N1 virus outbreaks more effectively in Guangdong. From the above, it was necessary to conduct continuously epidemiological survey and study the pathogenicity and antigenic variation of avian influenza in Southern China.

Keywords: H5N1, pathogenicity, transmissibility, antigenic variation, vaccine 


\section{INTRODUCTION}

Influenza $A$ viruses are the most important pathogen of three types (A, B, and $\mathrm{C}$ ) of influenza viruses, to both the poultry industry and human health. To date, avian influenza viruses representing $16 \mathrm{HA}$ and $9 \mathrm{NA}$ subtypes have been detected in wild birds and poultry throughout the world (Webster et al., 1992; Fouchier and Munster, 2009). According to the virulence of viruses, avian influenza viruses are divided into highly pathogenic avian influenza virus (HPAIV), low pathogenic avian influenza virus (LPAIV), and non-pathogenic avian influenza virus (NPAIV). However, only some influenza viruses of $\mathrm{H} 5$ and H7 subtype are highly pathogenic to poultry.

The H5N1 HPAIV was first isolated in Guangdong, China in 1996 (Xu et al., 1999). In 1997, H5N1 HPAIVs had repeatedly caused serious outbreaks among poultry farms and markets in Hong Kong, which resulted in heavy losses. And it was the first report that H5N1 HPAIV infected human in "Hong Kong Flu" in 1997, causing six deaths in 18 infection cases (Claas et al., 1998; Subbarao et al., 1998). In 2002, a new H5N1 HPAI outbreak in Hong Kong infected millions of birds, including several types of wild water fowl. This was the first time the H5N1 HPAIV was found to infect water fowl (Lee et al., 2005; Nguyen et al., 2005). Between 2003 and 2005, the H5N1 HPAI repetitively broke out in East Asia and South Asia, and even spread to Europe and Africa. This resulted in more than 150 million birds dead or slaughtered and 53 human fatalities (SturmRamirez et al., 2004; World Health Organization, 2005). Since 2003, the H5N1 HPAI had continued influencing more than 60 states or areas including Laos, Vietnam, Thailand, Hong Kong, and China (World Health Organization, 2013b). From 2003 to 1 May 2015, 840 laboratory-confirmed human cases of H5N1 HPAIVs infection were officially reported to WHO from 16 countries; of these cases, 447 died (World Health Organization, 2015). Consequently, H5N1 HPAIVs are zoonotic etiological agents recognized as a severe threat to both the poultry industry and human public health around the world.

In terms of antigenic characteristics, H5N1 HPAIVs were divided into 10 clades (0-9) and numerous subclades by World Health Organization/World Organization for Animal Health/Food and Agriculture Organization H5N1 Evolution Working Group (2008) ${ }^{1}$. Complicated breeding environments, the long distance transport of live poultry, and wild bird migration resulted in all known clades circulating endlessly in poultry in China. Especially during 2005 and 2006, H5N1 viruses of clades $2.2,2.3 .2,2.3 .4,4,7$, and 9 circulated all over China. Since 2007, viruses of clades 2.3.2, 2.3.4, and 7 have predominantly co-circulated continuously in domestic poultry and waterfowl in China (Smith et al., 2009; Jiang et al., 2010; Li et al., 2010). Later, several studies results showed that the pathogenicity of clade 2.3.2 viruses were intensifying in aquatic birds (Sakoda et al., 2010). Viruses of clade 7 began spreading in chickens across the northern of China in 2005, which had a high pathogenicity in chickens, but only a

${ }^{1}$ Food and Agricultural Organization(FAO). H5N1 HPAI Global Overview - July and August 2010, prepared by EMPRESS/GLEW, Issues No. 24. few viruses were isolated from aquatic birds. In 2008, H5N1 HPAI caused clade 7.2 viruses broke out in several cities in North China and caused a considerable amount of deaths in poultry. In 2010, a new H5N1 HPAIV belonging to clade 2.3.2.1 was isolated from South Asia, and 48 humans were reported to have been infected with the virus (Reid et al., 2011). In 2011, the H5N1 HPAI caused clade 2.3.2.1 viruses broke out in crows (World Health Organization, 2012). From 2012 to 2013, the H5N1 HPAIVs belonging to 2.3.2.1, 2.3.4, and 7.2 clades were detected in birds and/or environmental samples in China (World Health Organization, 2013a), but the most isolates belonged to clade 2.3.2.1. The pathogenicity of different clades varied in poultry and wild birds, but the movement and interaction of $\mathrm{H} 5 \mathrm{~N} 1$ viruses between them was still not clear until now.

To better understand the pathogenicity and transmissibility of different clade of $\mathrm{H} 5 \mathrm{~N} 1$ isolates from poultry in Guangdong in 2012, we selected two viruses-A/Chicken/China/G2/2012(H5N1) (G2) and A/Duck/China/G3/2012(H5N1) (G3)—to carry out their infection experiments. To evaluate the antigenic variation of these viruses and protective efficacy of current commercial vaccines against most isolates from Guangdong in 2012, G3 (belonging to clade 2.3.2.1) was chosen to challenge three commercial vaccines such as $\mathrm{Re}-4, \mathrm{Re}-6$, and D7.

\section{MATERIALS AND METHODS \\ H5N1 HPAIV Variants and Propagation}

The two H5N1 HPAIVs-A/Chicken/China/G2/2012(H5N1) (G2) and A/Duck/China/G3/2012(H5N1) (G3) - used in this study were isolated from cloacal swabs of apparently healthy birds in live bird markets during 2012. They were purified and propagated by three rounds of limiting dilution in the allantoic cavity of 9-11 days old specific-pathogen-free (SPF) embryonated chicken eggs (Jiao et al., 2014; Yuan et al., 2014). The allantoic fluid from multiple eggs was pooled, clarified by centrifugation, and frozen in aliquots at $-70^{\circ} \mathrm{C}$. The G2 and G3 inactivated antigens and positive serums were provided by College of Veterinary Medicine, South China Agricultural University. The $50 \%$ egg infectious dose (EID 50 ) was calculated according to the method published by Reed and Muench (1938) using the serial titration of eggs. All experiments were carried out in Animal Biosafety Level 3 (ABSL-3) facilities.

\section{Genetic and Phylogenetic Analyses}

The viral RNA was extracted from the allantoic fluid supernatant using Trizol LS Reagent (Life Technologies, Inc.). A reverse transcription polymerase chain reaction (RT-PCR) was conducted using Superscript III (Invitrogen, Carlsbad, CA, USA) and Unil2 (5-AGCAAAAGCAGG-3) primer. Eight genes were amplified using universal primers (Hoffmann et al., 2001), and the PCR products were purified using the mini PCR Purification Kit (Promega). Sequencing was performed by Shanghai Invitrogen Biotechnology Co., Ltd. The sequencing 
data were compiled with the Seqman program of Lasergene 7 (DNASTAR, Inc.). Amino acid sequence similarities were identified with the Lasergene 7 Megalign program (DNASTAR). The hemagglutinin (HA) gene phylogenetic tree of the $\mathrm{H} 5 \mathrm{~N} 1$ HPAIVs was created with MEGA 5 software (Sinauer Associates, Inc., Sunderland, MA).

The nucleotide sequences of A/Chicken/China/G2/ 2012(H5N1) (G2) and A/Duck/China/G3/2012(H5N1) (G3) were available from GenBank under the accession numbers KU851866-KU851867.

\section{Pathogenicity and Transmission}

Five-week-old SPF White Leghorn chickens were purchased from Beijing Merial Vital Laboratory Animal Technologies Co., LTD, Beijing, China.

To determine the pathogenicity and transmission of the two H5N1 HPAIVs, twenty-seven chickens were equally divided into three groups G2, G3, and control. Six chickens of G2 and G3 group were inoculated intranasally with $10^{3} \mathrm{EID}_{50}$ of G2 or G3 viruses, respectively; the other three chickens of each group were inoculated intranasally with the same volume of phosphate buffered saline (PBS), as naive contact housed with the inoculated chickens. The chickens of control group were inoculated intranasally with the same volume of PBS. All chickens were observed for clinical symptoms for 14 days. Three inoculated chickens in each group were euthanized at 3 days postinoculation (DPI), and the lungs, kidneys, liver, heart, spleen, and brain were collected. Similar executions were performed on chickens that died during the observation. Oropharyngeal and cloacal swabs were collected from all chickens at 3, 5, 7, 9, and $11 \mathrm{DPI}$, and suspended in $1 \mathrm{ml}$ isolation media PBS ( $\mathrm{pH} \mathrm{7.4).}$ All of the tissues and swabs were collected and titrated for virus infectivity in eggs, as described previously (Chen et al., 2004; Jiao et al., 2008). Seroconversion of the surviving chickens on 14 DPI was confirmed by hemagglutinin inhibition (HI) test. HI titers of the serums were detected using 1\% chicken red blood cells by a standard method (Takatsy and Barb, 1973). All animal experiments were conducted under the guidance of SCAU's Institutional Animal Care and Use Committee. Our animal experiments in this study had been approved by SCAU and were carried out in high-efficiency particulate airfiltered isolators (size: $2200 \times 850 \times 1700 \mathrm{~mm}$ ) and ABSL-3 facilities.

\section{Vaccine-Challenge}

To evaluate the antigenic variation of these viruses and protective efficacy of current commercial vaccines against most isolates from Guangdong in 2012, 3-week-old SPF White Leghorn chickens were purchased from Beijing Merial Vital Laboratory Animal Technologies Co., LTD, Beijing, China. Re-4 and Re-6 vaccines strain inactivated antigens, positive serums, and vaccines were purchased from Weike Biotechnology Co., Ltd., Harbin, China. D7 (H5N2) vaccines strain inactivated antigens, positive serums, and vaccines were purchased from Guangzhou South China Biological Medicine Co. Ltd., Guangdong, China.

Thirty-six chickens were divided into four groups $(n=9)$, and three groups were immunized with $0.3 \mathrm{ml}$ of Re- 4 , Re6 , or D7 inactivated vaccines via intramuscular injection, the control group received $0.3 \mathrm{ml}$ of PBS intramuscularly. Serum was collected from every chicken on 14 and 28 day-post-vaccination (DPV) for HI titers determination.

At $28 \mathrm{DPV}$, chickens were intranasally challenged with 200ul $10^{6} \mathrm{EID}_{50}$ of A/Duck/China/G3/2012(H5N1) (G3). Oropharyngeal and cloacal swabs were taken on days 3, 5, 7, 9, and 11 post-challenge, including chickens that died during this period. All swabs were immediately suspended in $1 \mathrm{ml}$ isolation media PBS, which were inoculated into 9-10 days old embryonated chicken eggs for examination of virus shedding. All surviving chickens were observed for clinical symptoms for 14 days and collected serum for seroconversion detection in the end.

\section{RESULTS}

\section{Genetic and Phylogenetic Analysis}

The HA genes of each virus were sequenced to determine the molecular evolution of the two viruses. The sequences were compared with representative $\mathrm{H} 5 \mathrm{~N} 1$ sequences obtained from GenBank. According to antigenic characteristics by the WHO, the HA gene of G2 belonged to clade 7.2, and that of G3 belonged to clade 2.3.2.1 (Figure 1). Their HA genes had a series of basic amino acids at the cleavage site of the HA (-RRRKR/GLF-), which represents the high pathogenicity of the H5N1 AIVs in poultry (Gohrbandt et al., 2011).

The amino acid sequences of the two viruses revealed five conservative potential N-linked glycosylation sites in HA (26, 27, 39, 499, and 558): three in HA1 $(26,27$, and 39) and two in

TABLE 1 | Cleavage site and potential glycosylation sites in HA of the two H5N1 HPAIVs (A/Chicken/China/G2/2012(H5N1) = G2 and A/Duck/China/G3/2012(H5N1) = G3).

\begin{tabular}{|c|c|c|c|c|c|c|c|c|c|c|c|c|}
\hline \multirow[t]{3}{*}{ Strains } & \multirow{2}{*}{$\begin{array}{l}\text { Cleavage site } \\
\qquad 342-347\end{array}$} & \multicolumn{11}{|c|}{ potential glycosylation sites } \\
\hline & & 26 & 27 & 39 & 155 & 169 & 178 & 209 & 251 & 301 & 499 & 558 \\
\hline & -RRRKR/G- & NNS & NST & NVT & NSS & NNT & NNT & NLT & NDT & NSS & NGT & NGS \\
\hline G2 & $+^{a}$ & + & + & + & NPS & + & $-^{b}$ & - & + & + & + & + \\
\hline G3 & + & + & + & + & + & - & + & - & - & NYS & + & + \\
\hline
\end{tabular}

a The "+" means the amino acid sequences of glycosylation sites are same with list above.

bThe "-" means the glycosylation sites are lost. 
HA2 (499 and 558). In addition, the G2 virus lost two potential $\mathrm{N}$-linked glycosylation sites in 178 (NNT) and 209 (NPT), and amino acids at 155 the glycosylation site changed from NSS to NPS (Table 4). The G3 HA lost three potential N-linked

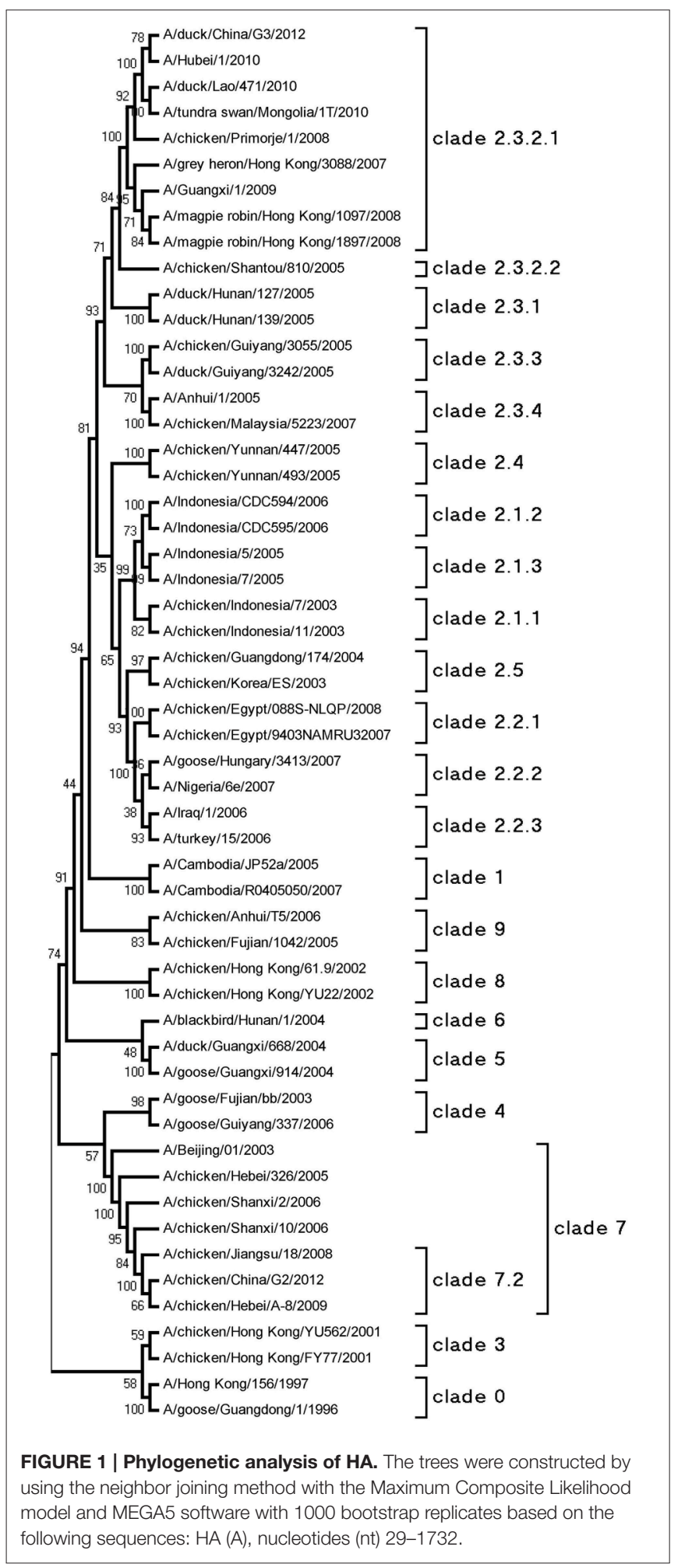

glycosylation sites in 169 (NNT), 209 (NPT) and 251 (NDT), and amino acids at 301 the glycosylation site changed from NSS to NYS (Table 1).

\section{Pathogenicity of H5N1 HPAIVs in Chickens}

To evaluate the pathogenicity of the two H5N1 HPAIVs, six chickens of each group were inoculated intranasally with 100 $\mu 110^{3}$ EID $_{50} \mathrm{G} 2, \mathrm{G} 3$, or PBS, respectively. All chickens in the G3 group began to show clinical typical symptoms as early as two DPI, and were dead by four DPI (Figure 2). However, the inoculated chickens of the G2 group showed clinical signs by four DPI, and all died by eight DPI (Figure 2). So the lethality of G2 and $\mathrm{G} 3$ viruses in chickens was $100 \%$ (Table 2).

Eyelid edema, insensibility, diminished appetite and thirst, roughened hair coats, comb cyanosis, torticollis, ataxia, and other neurological symptoms were observed among dead chickens infected with G2 and G3. At necropsy of chickens dead from infection we found slight petechial hemorrhaging in subcutaneous fat, hyperaemia, haemorrhagia, and nignecrosis in the lungs; hepatomegaly and an amber liver; and hyperaemia and haemorrhagia in the stomachus glandularis. In short, both G2 and G3 viruses produced apparent clinical symptoms and typical pathological changes in severe infected chickens.

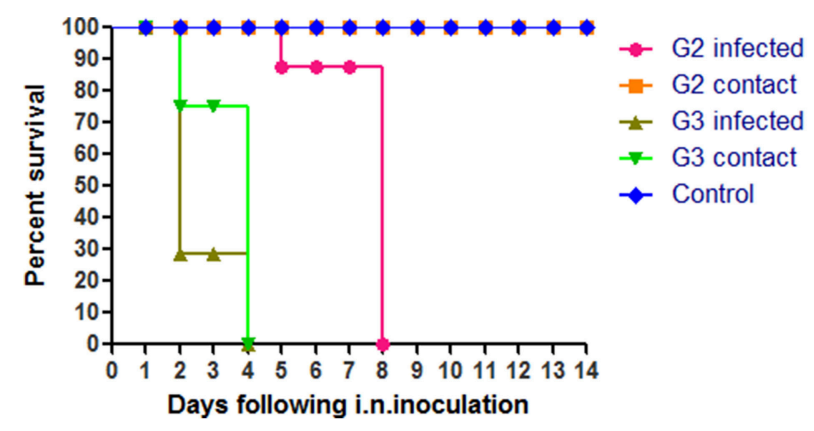

FIGURE 2 | Lethality of the G2 and G3 viruses in SPF chickens. The G2 infected chickens were inoculated intranasally with $100 \mu l 10^{3} \mathrm{EID}_{50} \mathrm{G} 2$ viruses and the G2 contact chickens were housed with them without inoculate. The G3 infected chickens were inoculated intranasally with 100 ul $10^{3} \mathrm{EID}_{50}$ G3 viruses and the G3 contact chickens were housed with them without inoculate.

TABLE 2 | Clinical situations and lethality of chickens after inoculated intranasally with the two H5N1 HPAIVs (A/Chicken/China/G2/2012 $($ H5N1) $=$ G2 and A/Duck/China/G3/2012(H5N1) = G3).

\begin{tabular}{|c|c|c|c|c|}
\hline \multicolumn{2}{|l|}{ Strains } & \multirow{2}{*}{$\begin{array}{c}\text { Clinical symptom } \\
\text { rates } \\
100 \%\end{array}$} & \multirow{2}{*}{$\begin{array}{c}\text { Virus shedding } \\
\text { proportions } \\
100 \%\end{array}$} & \multirow{2}{*}{$\frac{\text { Survival }}{}$} \\
\hline G2 & Infected ${ }^{\mathrm{a}}$ & & & \\
\hline & Contact $^{\mathrm{b}}$ & $100 \%$ & 0 & $100 \%$ \\
\hline \multirow[t]{2}{*}{ G3 } & Infected & $100 \%$ & $100 \%$ & 0 \\
\hline & Contact & $100 \%$ & $100 \%$ & 0 \\
\hline Control & & 0 & 0 & $100 \%$ \\
\hline
\end{tabular}

${ }^{a}$ Chickens inoculated with virus.

${ }^{b}$ Contact chickens housed with those inoculated. 
To evaluate the replication of the two viruses in chickens, three inoculated chickens in each group were euthanized at three DPI, and the lungs, kidneys, liver, heart, spleen, and brain were collected. Oropharyngeal and cloacal swabs were collected from chickens of each group at 3, 5, 7, 9, and 11 DPI. All of the tissues and swabs were collected and titrated for virus infectivity. In G2 inoculated chickens, the virus replicated in all tested organs on three DPI and the mean titers were $4.33 \log \mathrm{EID}_{50}$ in the heart, $2.92 \log \mathrm{EID}_{50}$ in the liver, $2.67 \log \mathrm{EID}_{50}$ in the spleen, $1.58 \log$ EID $_{50}$ in the lungs, $3.67 \log \mathrm{EID}_{50}$ in the kidneys, and $3.25 \mathrm{log}$ $\mathrm{EID}_{50}$ in the brain (Table 3). In G3 virus inoculated chickens, the virus replicated to higher and the mean titers were $5.25 \log$ EID $_{50}$ in the liver and $5.5 \log \mathrm{EID}_{50}$ in the heart, spleen, lungs, kidneys, and brain, respectively (Table 3 ). Above all, the replication of G3 in chicken was much higher than that of G2.

In the G2 group, virus shedding was detected from the oropharynx and cloaca swabs in inoculated chickens within seven DPI. The virus titers from oropharynx swabs and cloacal swabs were 2.92 and $1.96 \log \mathrm{EID}_{50}, 2.63$ and $1.88 \log \mathrm{EID}_{50}$, and 2.63 and $1.63 \log \mathrm{EID}_{50}$ on three DPI, five DPI, and seven DPI, respectively (Table 4). All of the chickens in the G2 group died within eight DPI. G3 virus shedding could be tested from both oropharyngeal and cloacal swabs in inoculated chickens on three DPI, and the virus titers were all $4.5 \log \mathrm{EID}_{50}$ (Table 4). All of the chickens in the G3 group died within four DPI. These showed that the duration of virus shedding of chickens infected with G2 was 8 days and was longer than the 4 days of G3, but the titers of replication of G2 group were lower than that of G3. Therefore, our results indicated that both G2 and G3 were highly pathogenic to chickens, and the replication of the G3 virus was higher than that of the G2.

\section{Transmission of H5N1 HPAIVs in Chickens}

To understand the naive contact transmission of these two viruses, three SPF chickens were inoculated intranasally with $0.1 \mathrm{ml}$ PBS as naive control group and housed with inoculated chickens of the G2 and G3 groups, respectively. Oropharyngeal and cloacal swabs were collected from them at 3, 5, 7, 9, and 11 DPI. All surviving chickens were observed for 14 days. We collected and titrated the tissues and swabs for virus infectivity.

During the observation period, the naive contact chickens in the G2 group began to show mild clinical signs, such as spirits atrophy and inappetence, by five DPI and these mild clinical signs disappeared by seven DPI, all chickens survived for 14 days and

TABLE 3 | Replication of the two H5N1 HPAIVs (A/Chicken/China/G2/2012(H5N1) = G2 and A/Duck/China/G3/2012(H5N1) = G3) in SPF chickens ${ }^{a}$.

\begin{tabular}{|c|c|c|c|c|c|c|c|}
\hline \multirow[t]{2}{*}{ Strains } & & \multicolumn{6}{|c|}{ Virus titers in SPF chickens at 3 DPI $\left(\log _{10} E_{1 D_{50}} / 0.1 \mathrm{ml}\right)$ in } \\
\hline & & Heart & Liver & Spleen & Lungs & Kidneys & Brain \\
\hline \multirow[t]{2}{*}{ G2 } & Infected & $4.33 \pm 0.52$ & $2.92 \pm 0.52$ & $2.67 \pm 0.14$ & $1.58 \pm 0.14$ & $3.67 \pm 0.52$ & $3.25 \pm 0.5$ \\
\hline & Contact & 0 & 0 & 0 & 0 & 0 & 0 \\
\hline \multirow[t]{2}{*}{ G3 } & Infected & $5.5 \pm 0$ & $5.25 \pm 0.43$ & $5.5 \pm 0$ & $5.5 \pm 0$ & $5.5 \pm 0$ & $5.5 \pm 0$ \\
\hline & Contact & $5.5 \pm 0$ & $4.75 \pm 0.66$ & $5 \pm 0.66$ & $4.83 \pm 0.63$ & $5.25 \pm 0.43$ & $5.0 \pm 0.43$ \\
\hline Control & & 0 & 0 & 0 & 0 & 0 & 0 \\
\hline
\end{tabular}

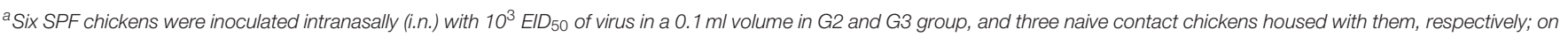

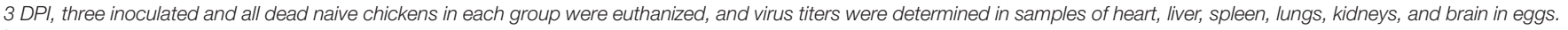

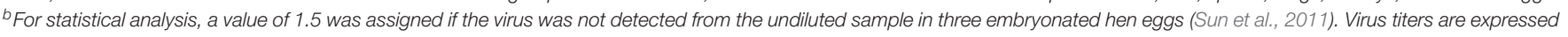
as means \pm standard deviation in $\log 10 E / D_{50} / 0.1 \mathrm{ml}$ of tissue.

TABLE 4 | Virus titers in oropharyngeal and cloacal swabs from chickens after inoculated with the two H5N1 HPAIVs (A/Chicken/China/G2/2012(H5N1) = G2 and A/Duck/China/G3/2012(H5N1) = G3).

\begin{tabular}{|c|c|c|c|c|c|c|c|c|c|}
\hline \multirow[t]{3}{*}{ Strains } & & \multicolumn{8}{|c|}{ Days post-inoculation $\left(\log _{10} \mathrm{EID}^{50} / 0.1 \mathrm{ml}\right) \pm \mathrm{SD}^{\mathrm{a}}$} \\
\hline & & \multicolumn{2}{|c|}{3 day } & \multicolumn{2}{|c|}{5 day } & \multicolumn{2}{|c|}{7 day } & \multicolumn{2}{|l|}{9 day } \\
\hline & & $\begin{array}{c}\text { Oropharyngeal } \\
\text { swabs }\end{array}$ & $\begin{array}{l}\text { Cloacal } \\
\text { swabs }\end{array}$ & $\begin{array}{c}\text { Oropharyngeal } \\
\text { swabs }\end{array}$ & $\begin{array}{l}\text { Cloacal } \\
\text { swabs }\end{array}$ & $\begin{array}{c}\text { Oropharyngeal } \\
\text { swabs }\end{array}$ & $\begin{array}{l}\text { Cloacal } \\
\text { swabs }\end{array}$ & $\begin{array}{l}\text { Oropharyngeal } \\
\text { swabs }\end{array}$ & $\begin{array}{r}\text { Cloacal } \\
\text { swabs }\end{array}$ \\
\hline \multirow[t]{2}{*}{ G2 } & Infected ${ }^{b}$ & $\begin{array}{c}2.92 \pm 1.11 \\
(5 / 6)\end{array}$ & $\begin{array}{c}1.96 \pm 0.46 \\
(5 / 6)\end{array}$ & $\begin{array}{c}2.63 \pm 0.18 \\
(2 / 2)\end{array}$ & $\begin{array}{c}1.88 \pm 0.53 \\
(1 / 2)\end{array}$ & $\begin{array}{c}2.63 \pm 0.18 \\
(2 / 2)\end{array}$ & $\begin{array}{c}1.63 \pm 0.18 \\
(1 / 2)\end{array}$ & $N D^{d}$ & ND \\
\hline & Contact $^{\mathrm{C}}$ & 0 & 0 & 0 & 0 & 0 & 0 & 0 & 0 \\
\hline \multirow[t]{2}{*}{ G3 } & Infected & $4.5 \pm 0(1 / 1)$ & $4.5 \pm 0(1 / 1)$ & ND & - & - & - & - & - \\
\hline & Contact & $4.5 \pm 0(2 / 2)$ & $4.5 \pm 0(2 / 2)$ & ND & - & - & - & - & - \\
\hline Control & & 0 & 0 & 0 & 0 & 0 & 0 & 0 & 0 \\
\hline
\end{tabular}

${ }^{a}$ For statistical purposes, a value of 1.5 was assigned if virus was not detected from the undiluted sample in three embryonated hen's eggs (Sun et al., 2011).

${ }^{b}$ Chickens inoculated with virus.

${ }^{c}$ Contact chickens housed with those inoculated.

${ }^{d} N D$ : not detected. Chickens all died. 
the seroconversion rate was $100 \%$. The naive contact chickens in the G3 group began to show clinical signs by two DPI and all died by four DPI (Figure 2). In the G3 naive contact group, virus replication titers were $5.5 \log \mathrm{EID}_{50}$ in the heart, $4.75 \log$ $\mathrm{EID}_{50}$ in the liver, $5 \log \mathrm{EID}_{50}$ in the spleen, $4.83 \log \mathrm{EID}_{50}$ in the lungs, $5.25 \log \mathrm{EID}_{50}$ in the kidneys, and $5 \log \mathrm{EID}_{50}$ in the brain (Table 3). However, no virus was detected in tissue samples of the G2 naive contact group. All of the naive contact chickens in G3 group could shed virus from the oropharynx and cloaca swabs; the virus titers were both $4.5 \log \mathrm{EID}_{50}$ at three DPI, which was the same as that of inoculated chickens (Table 4). In the chickens of the G2 naive contact group, virus shedding could not be detected all along (Table 4). These showed that the lethality of naive contact chickens of G3 was $100 \%$ and that of G2 was 0 although both G2 and G3 had naive contact transmission in chickens (Table 2). So G3 virus had stronger transmissibility between chickens by naive contact than G2.

\section{Antigenic Variation of HPAIV and Protective Efficacy of Current Commercial Vaccines}

To characterize antigenic variation of the two H5N1 HPAIVs and the current commercially vaccines strains, we carried out the cross reactive $\mathrm{HI}$ assay. The cross reactive $\mathrm{HI}$ antibody titers of anti-Re-4, anti-Re-6, and anti-D7 serum in reaction with G2 antigen were $5 \log 2,1 \log 2$, and $2 \log 2$, respectively; and those in reaction with G3 antigen were $5 \log 2,6 \log 2$, and $9 \log 2$, respectively (Table 5). Our results showed that the antigens of G2 and G3 were very different from those of vaccine isolates. Therefore, we should assess the immunogenicity and effectiveness of three current commercially inactivated vaccines against these isolates in 2012 in Guangdong of China.

Because H5N1 HPAIVs of clade 2.3.2.1 have most isolates from Guangdong in 2012, we estimated the effectiveness of current commercial vaccines against G3. Three-week-old SPF chickens were immunized with inactivated vaccines such as Re-4, Re-6, and D7, respectively. Serum from every group was collected at 14 and $28 \mathrm{DPV}$ for HI test, respectively. Then, all chickens at $28 \mathrm{DPV}$ were challenged intranasally with $200 \mu 11^{6} \mathrm{EID}_{50}$ of G3. In the Re-4 group, chickens were challenged with G3 when the mean HI titer was $9.4 \log 2$ at $28 \mathrm{DPV}$. The chickens began to show clinical symptoms on three DPI and die on six DPI, shedding virus was tested on three to nine DPI, and the mortality and virus shedding proportion were 33.3 and $88.9 \%$, respectively (Table 6). In the Re-6 group, chickens were challenged when the mean HI titer was $7.0 \log 2$ at $28 \mathrm{DPV}$. The chickens began to die on six DPI, virus shedding was detected on three to eleven DPI, and the mortality and virus shedding proportion were 11.1 and $44.4 \%$, respectively (Table 6). Those results showed that the Re-6 vaccine has a certain degree of protection against the $\mathrm{G} 3$ virus. In the D7 group, chickens were challenged when the mean HI titer was $7.4 \log 2$ at $28 \mathrm{DPV}$, and no virus shedding or death was found during the observation period (Table 6). In the non-immunized control group, the HI titer was zero. All chickens were detected virus shedding and died during the observation period. These findings showed that the protection rate of the $\mathrm{D} 7$ vaccine against G3 was $100 \%$, that of Re- 6 was $88.9 \%$, and Re- 4 was $66.7 \%$.
TABLE 5 | Cross reactive hemagglutination inhibition (HI) ${ }^{\mathrm{a}}$ antibody titers of anti-serum against five avian influenza virus antigens.

\begin{tabular}{lcccccc}
\hline Strains & Clade & \multicolumn{5}{c}{ Serum of HI titers (log2) } \\
\cline { 3 - 6 } & & Re-4 & Re-6 & D7 & G2 & G3 \\
\hline Re-4 & 7 & 10 & 3 & 8 & 2 & 5 \\
Re-6 & 2.3 .2 & 5 & 8 & 10 & 1 & 10 \\
D7 & 2.3 .2 & 5 & 6 & 9 & 1 & 9 \\
G2 & 7.2 & 5 & 1 & 2 & 8 & 5 \\
G3 & 2.3 .2 .1 & 5 & 6 & 9 & 0 & 10 \\
\hline
\end{tabular}

a The cross reactive $\mathrm{HI}$ assays were carried out according to WHO standard method.

TABLE 6 | Results of hemagglutination inhibition (HI) titers from serum samples of chickens at 28 DPV. And the protection rates of three vaccines against G3 virus challanged ${ }^{\mathrm{a}}$.

\begin{tabular}{|c|c|c|c|}
\hline \multirow[t]{2}{*}{ Groups } & \multirow{2}{*}{$\begin{array}{c}\text { Before challenged } \\
\text { HI antibody titers } \\
(\log 2)\end{array}$} & \multirow{2}{*}{$\begin{array}{c}\text { Post-challenge } \\
\text { Viruses } \\
\text { shedding (\%) }\end{array}$} & \multirow[t]{2}{*}{ Survival rate (\%) } \\
\hline & & & \\
\hline $\mathrm{Re}-4$ & $9.40^{C}$ & 88.9 & 66.7 \\
\hline Re-6 & 7.20 & 44.4 & 88.9 \\
\hline D7 & 7.50 & 0 & 100 \\
\hline Control & 0 & 100 & 0 \\
\hline
\end{tabular}

aThirty-six three-week-old SPF chickens were divided into four groups and immunized with inactivated Re-4, Re-6, D7, and PBS respectively. At 28 DPV, all chickens were challenged intranasally with $200 \mathrm{ul} 10^{6}$ EID 50 of G3.

${ }^{b}$ Serum samples from Re-4 group, Re-6 group, and D7 group were detected with Re-4, Re-6, and D7 inactivated antigens, respectively. Serum samples from control group were detected simultaneously with Re-4, Re-6, and D7 inactivated antigens.

${ }^{c}$ Geometric mean titer (GMT).

In a word, the mean $\mathrm{HI}$ titers in all immune groups were higher than $6 \log 2$, which indicated that these three commercial vaccines (Re-4, Re-6, and D7) had good immunogenicity in chickens. And the results of challenge study showed that these vaccines gave certain protection against G3, but their protection rates were different. Combined with the results of the cross reactive $\mathrm{HI}$ assay, we found that some vaccine strains were not antigenically well-matched with epidemic isolates, so the protective effects of the three vaccines varied.

\section{DISCUSSION}

The first H5N1 HPAIV in China was isolated from sick geese in Guangdong province in 1996 (Xu et al., 1999). In the following years, H5N1 HPAIVs repeatedly caused serious outbreaks in South China, especially in Hong Kong, and resulted in heavy losses of economy and life. Most of H5N1 viruses rapidly spread and induced large numbers of death within 2 or 3 days in chickens. The ducks and geese infected H5N1 HPAIVs showed no clinical symptoms in the past, but new H5N1 HPAIVs could attacked ducks and/or geese and caused deaths in recent years ( $\mathrm{Li}$ et al., 2010). In addition, more and more mammal was susceptible to H5N1 HPAIV by natural or laboratory infections. Felines, including cat, tiger, lion, leopard, clouded leopard, and Asiatic 
golden cat were highly susceptible to H5N1 HPAIV (Reperant et al., 2009). The domestic dog, hamster, rhesus macaque, cynomolgus, palm civet, red fox and raccoon could be potentially fatal by H5N1 HPAIV. Pika, domesticated swine, cattle, donkey, rat, and rabbit can exhibit asymptomatic or nonfatal infections by H5N1 HPAIV (U.S. Geological Survey, 2011). From 2010 to 2013 the dominant clades of H5N1 HPAIVs co-circulating in South China were 2.3.2.1 and 7.2 although other clades, such as 2.3.4, had occasionally been detected (World Health Organization, 2013a). In our study, the G2 and G3 strains from poultry in Guangdong in 2012 belonged to clades 7.2 and 2.3.2.1, respectively. The inoculation dose was mostly $10^{6} \mathrm{EID}_{50}$ or $10^{5}$ $\mathrm{EID}_{50}$ in previous pathogenicity studies, but here we selected a medium infective dose $\left(10^{3} \mathrm{EID}_{50}\right)$ to observe the difference of pathogenicity and transmission of the H5N1 HPAIVs. This might be one of the reasons why the naive contact chickens in G2 group showed mild clinical symptoms without any viruses shedding or death. In our study, both G2 and G3 virus could highly replicated in the heart, liver, brain, spleen, kidneys, and lungs of infected chickens, virus shedding could be detected from all infected chickens during survival, and the lethal rates were both $100 \%$. These results showed that G2 and G3 virus had high pathogenicity to chickens. By this token, the new H5N1 HPAIVs of these two clades in South China still had high pathogenicity to chickens.

AIV could form an aerosol and horizontal transmission through the respiratory tract in poultry. In recent years, the dominant AIVs co-circulated in mainland China were $\mathrm{H} 5$ and H9 subtypes, of which H9 subtype AIVs have strong horizontal transmission. However, only some H5 AIVs had horizontal transmission ability (World Health Organization, 2013a). In our previous studies, some H5N1 AIVs, which belonged to clades 0 , 2.3.2.2, 7.2, and 9, could horizontally transmit between chickens, ducks, geese, Japanese quails, and mice (Sun et al., 2011). Here, G2 and G3 virus belonged to clades 7.2 and 2.3.2.1, respectively, and both of them could transmit horizontally in chickens. All of the naive contact chickens in the G3 group had detected viruses shedding and replication in organs, but the naive contact chickens in the G2 group only had mild clinical symptoms and had no death or virus shedding. These results showed the new H5N1 HPAIVs of these two clades had different horizontal transmission ability. Moreover, H5N1 HPAIV of clade 2.3.2.1 still have been circulating in poultry and wild bird up to now, so they will continue to have the threat to human health and poultry product.

In China, poultry production modes, including rural household scatter breeding, poultry farms, and modern poultry ranches, are multiple and complicated so that prevention and control of H5 HPAI are difficult. Therefore, all poultry in China were immunized with $\mathrm{H} 5 \mathrm{~N} 1$ vaccinations compulsorily. In recent decades, several $\mathrm{H} 5$ vaccines, especially $\mathrm{H} 5$ inactivated vaccines, were widely used in China due to constant mutation and evolution of the virus (Swayne, 2012). The first commercial flu vaccine in China was an H5N2 inactivated vaccine, which used the low pathogenic avian influenza H5N2 virus A/Turkey/England/N-28/1973 and was approved for use in August of 2003 (Chen and $\mathrm{Bu}, 2009$ ).
Then the Re-1 vaccine was approved for use in 2004, for which was antigenically well-matched the epidemic strains at that time. In 2006, the H5N1 Re-4 vaccine, whose strain belonged to clade 7, was approved into service in China and widely used in the northern mainland. In 2008, new Re-5 vaccine began to be used in northern and southern China, whose strain A/duck/Anhui/1/2006(H5N1) belonged to clade 2.3.4 (Jiang et al., 2010; Li et al., 2010). In 2012, the recombinant vaccine Re-6 was also approved for use in the mainland to control new epidemic strains in clade 2.3.2. In 2013, a new $\mathrm{H} 5$ vaccine $\mathrm{D} 7$ was approved for use in waterfowl, which used an H5N2 virus (A/duck/Guangdong/D7/2007) belonged to clade 2.3.2. In conclusion, although flu vaccines were updated constantly, new strains still continue to appear in China. Therefore, it is necessary to evaluate the effectiveness and effects of current vaccines against the new strains timely.

From 2011 to 2012, most H5 isolates circulating in Guangdong province belonged to clade 2.3.2.1, including G3, so we wanted to estimate the effectiveness of current vaccines against them. In our study, the effectiveness of these three commercial vaccines against G3 varied. The D7 vaccine provided $100 \%$ protection to chickens against G3, the Re-6 vaccine provided $88.9 \%$ protection, and the Re-4 vaccine only provided $66.7 \%$. The antibody titer of Re-4 in chicken had more $2 \log 2$ than Re6 and D7 when challenged at $28 \mathrm{DPV}$, but the protection rate of Re-4 against G3 was lowest because the Re-4 vaccine strain and G3 belonged to different clades. These results indicated that $\mathrm{Re}-4$ vaccine did not protect chickens against $\mathrm{H} 5$ viruses challenging although had good immunogenicity and could induce high antibody levels. The D7 vaccine provided the best protection in these three vaccines against G3, whose strain belonged to clade 2.3.2. These told us that high antibody levels did not provide good protection, what need antigen matching between vaccine and epidemic strains. Therefore, to evaluate vaccines more objectively and effectively, we should be concerned not only about antibody level of immunized animals but also antigen matching between vaccine strains and epidemic isolates when observed the protection of vaccines in clinical practices.

\section{AUTHOR CONTRIBUTIONS}

Conceived and designed the experiments: PJ, ML. Performed the experiments: XL, HS, YS, JC, NQ. Analyzed the data: PJ, HS, XL. Contributed reagents/materials/analysis tools: PJ, XL, YS, JC, JY, SW, NQ, TZ. Wrote the paper: HS, PJ. All authors read and approved the final manuscript.

\section{ACKNOWLEDGMENTS}

This work was supported by grants from the National Natural Science Foundation of China (No. U1501212 and 31172343), the Program of International Science and Technology Cooperation of China (No. 2013DFA31940) the Science and Technology Projects of Guangdong Province (No. 2012B020306003, 
2012A020100001 and 2014A050503061), the Science and Technology Projects of Guangzhou City (No. 201300000037

\section{REFERENCES}

Chen, H., and $\mathrm{Bu}, \mathrm{Z}$. (2009). Development and application of avian influenza vaccines in China[J]. Curr. Top. Microbiol. Immunol. 333, 153-162. doi: 10.1007/978-3-540-92165-3 7

Chen, H., Deng, G., Li, Z., Tian, G., Li, Y., Jiao, P., et al. (2004). The evolution of $\mathrm{H} 5 \mathrm{~N} 1$ influenza viruses in ducks in southern China. Proc. Natl. Acad. Sci. U.S.A. 101, 10452-10457. doi: 10.1073/pnas.0403212101

Claas, E. C., Osterhaus, A. D., van Beek, R., De Jong, J. C., Rimmelzwaan, G. F., Senne, D. A., et al. (1998). Human influenza A H5N1 virus related to a highly pathogenic avian influenza virus. Lancet 351, 472-477. doi: 10.1016/S01406736(97)11212-0

Fouchier, R. A., and Munster, V. J. (2009). Epidemiology of low pathogenic avian influenza viruses in wild birds[J]. Rev Sci Tech. 28, 49-58. doi: 10.20506/rst.28.1.1863

Gohrbandt, S., Veits, J., Hundt, J., Bogs, J., Breithaupt, A., Teifke, J. P., et al. (2011). Amino acids adjacent to the haemagglutinin cleavage site are relevant for virulence of avian influenza viruses of subtype H5. J. Gen. Virol. 92, 51-59. doi: 10.1099/vir.0.023887-0

Hoffmann, E., Stech, J., Guan, Y., Webster, R. G., and Perez, D. R. (2001). Universal primer set for the full-length amplification of all influenza A viruses. Arch. Virol. 146, 2275-2289. doi: 10.1007/s007050170002

Jiang, W. M., Liu, S., Chen, J., Hou, G. Y., Li, J. P., Cao, Y. F., et al. (2010). Molecular epidemiological surveys of H5 subtype highly pathogenic avian influenza viruses in poultry in China during 2007-2009. J. Gen. Virol. 91, 2491-2496. doi: 10.1099/vir.0.023168-0

Jiao, P., Tian, G., Li, Y., Deng, G., Jiang, Y., Liu, C., et al. (2008). A single-aminoacid substitution in the NS1 protein changes the pathogenicity of H5N1 avian influenza viruses in mice. J. Virol. 82, 1146-1154. doi: 10.1128/JVI.01698-07

Jiao, P., Wei, L., Song, Y., Cui, J., Song, H., Cao, L., et al. (2014). D701N mutation in the PB2 protein contributes to the pathogenicity of $\mathrm{H} 5 \mathrm{~N} 1$ avian influenza viruses but not transmissibility in guinea pigs. Front. Microbiol. 5:642. doi: 10.3389/fmicb.2014.00642

Lee, C. W., Suarez, D. L., Tumpey, T. M., Sung, H. W., Kwon, Y. K., Lee, Y. J., et al. (2005). Characterization of highly pathogenic H5N1 avian influenza A viruses isolated from South Korea. J. Virol. 79, 3692-3702. doi: 10.1128/JVI.79.6.36923702.2005

Li, Y., Shi, J., Zhong, G., Deng, G., Tian, G., Ge, J., et al. (2010). Continued evolution of $\mathrm{H} 5 \mathrm{~N} 1$ influenza viruses in wild birds, domestic poultry, and humans in China from 2004 to 2009. J. Virol. 84, 8389-8397. doi: 10.1128/JVI.00413-10

Nguyen, D. C., Uyeki, T. M., Jadhao, S., Maines, T., Shaw, M., Matsuoka, Y., et al. (2005). Isolation and characterization of avian influenza viruses, including highly pathogenic H5N1, from poultry in live bird markets in Hanoi, Vietnam, in 2001. J. Virol. 79, 4201-4212. doi: 10.1128/JVI.79.7.4201-4212.2005

Reed, L. J., and Muench, H. (1938). A simple method of estimating fifty per cent endpoints. Am. J. Epidemiol. 27, 493-497.

Reid, S. M., Shell, W. M., Barboi, G., Onita, I., Turcitu, M., Cioranu, R., et al. (2011). First reported incursion of highly pathogenic notifiable avian influenza A H5N1 viruses from clade 2.3.2 into European poultry. Transbound. Emerg. Dis. 58, 76-78. doi: 10.1111/j.1865-1682.2010.01175.x

Reperant, L. A., Rimmelzwaan, G. F., and Kuiken, T. (2009). Avian influenza viruses in mammals. Rev. Sci. Tech. 28, 137-159. doi: 10.20506/rst.28.1.1876

Sakoda, Y., Sugar, S., Batchluun, D., Erdene-Ochir, T. O., Okamatsu, M., Isoda, N., et al. (2010). Characterization of H5N1 highly pathogenic avian influenza virus strains isolated from migratory waterfowl in Mongolia on the way back from the southern Asia to their northern territory. Virology 406, 88-94. doi: 10.1016/j.virol.2010.07.007

Smith, G. J., Vijaykrishna, D., Ellis, T. M., Dyrting, K. C., Leung, Y. H., Bahl, J., et al. (2009). Characterization of avian influenza viruses A (H5N1) from wild birds, Hong Kong, 2004-2008. Emerg. Infect. Dis. 15, 402-407. doi: 10.3201/eid1503.081190

Sturm-Ramirez, K. M., Ellis, T., Bousfield, B., Bissett, L., Dyrting, K., Rehg, J. E., et al. (2004). Reemerging H5N1 influenza viruses in Hong Kong in 2002 are and 2013J4500030) and the Earmarked Fund for Modern Agro-Industry Technology Research System (CARS-42-G09). highly pathogenic to ducks. J. Virol. 78, 4892-4901. doi: 10.1128/JVI.78.9.48924901.2004

Subbarao, K., Klimov, A., Katz, J., Regnery, H., Lim, W., Hall, H., et al (1998). Characterization of an avian influenza A (H5N1) virus isolated from a child with a fatal respiratory illness. Science 279, 393-396. doi: $10.1126 /$ science. 279.5349 .393

Sun, H., Jiao, P., Jia, B., Xu, C., Wei, L., Shan, F., et al. (2011). Pathogenicity in quails and mice of $\mathrm{H} 5 \mathrm{~N} 1$ highly pathogenic avian influenza viruses isolated from ducks. Vet. Microbiol. 152, 258-265. doi: 10.1016/j.vetmic.2011. 05.009

Swayne, D. E. (2012). The role of vaccines and vaccination in high pathogenicity avian influenza control and eradication[J]. Expert Rev. Vaccines 11, 877-880. doi: 10.1586/erv. 12.60

Takatsy, G., and Barb, K. (1973). Antigenic analysis of prototype influenza a (H3N2) strains by the antiserum absorption method[J]. J. Hyg. (Lond.) 71, 501-508. doi: 10.1017/S0022172400046489

U.S. Geological Survey (2011). List of Species Affected by H5N1 (Avian Influenza). Reston, VA: U.S. Geological Survey-National Wildlife Health Center.

Webster, R. G., Bean, W. J., Gorman, O. T., Chambers, T. M., and Kawaoka, Y. (1992). Evolution and ecology of influenza A viruses. Microbiol. Rev. 56, 152-179.

World Health Organization (2005). Cumulative Number of Confirmed Human Cases of Avian Influenza A/(H5N1) Reported to WHO. Geneva: WHO. Available online at: www.who.int/csr/disease/avian/influenza/country/cases/table/2005/ 06/28/en/index.html

World Health Organization/World Organization for Animal Health/Food and Agriculture Organization H5N1 Evolution Working Group (2008). Toward a unified nomenclature system for highly pathogenic avian influenza virus (H5N1) (conference summary). Emerg. Infect.

World Health Organization (2012). Antigenic and Genetic Characteristics of Zoonotic Influenza Viruses and Development of Candidate Vaccine Viruses for Pandemic Preparedness. 2011-2012. Available online at: http://www.who.int/ influenza/vaccines/virus/characteristics_virus_vaccines/en/.

World Health Organization (2013a). Antigenic and Genetic Characteristics of Zoonotic Influenza Viruses and Development of Candidate Vaccine Viruses for Pandemic Preparedness. 2012-2013. Available online at: http://www.who.int/ influenza/vaccines/virus/characteristics_virus_vaccines/en/

World Health Organization (2013b). Cumulative Number of Confirmed Human Cases for Avian Influenza A(H5N1) Reported to WHO, 2003-2013. Available online at: http://www.who.int/influenza/human_animal_interface/EN_GIP_ 20130829CumulativeNumberH5N1cases

World Health Organization (2015). Influenza at the Human-Animal Interface. Summary and Assessment as of 1 May 2015. Available online at: http://www. who.int/influenza/human_animal_interface/Influenza

Xu, X., Subbarao, Cox, N.J., and Guo, Y. (1999). Genetic characterization of the pathogenic influenza A/Goose/Guangdong/1/96 (H5N1) virus: similarity of its hemagglutinin gene to those of H5N1 viruses from the 1997 outbreaks in Hong Kong. Virology 261, 15-19. doi: 10.1006/viro.19 99.9820

Yuan, R., Cui, J., Zhang, S., Cao, L., Liu, X., Kang, Y., et al. (2014). Pathogenicity and transmission of $\mathrm{H} 5 \mathrm{~N} 1$ avian influenza viruses in different birds. Vet. Microbiol. 168, 50-59. doi: 10.1016/j.vetmic.2013.10.013

Conflict of Interest Statement: The authors declare that the research was conducted in the absence of any commercial or financial relationships that could be construed as a potential conflict of interest.

Copyright (๑ 2016 Jiao, Song, Liu, Song, Cui, Wu, Ye, Qu, Zhang and Liao. This is an open-access article distributed under the terms of the Creative Commons Attribution License (CC BY). The use, distribution or reproduction in other forums is permitted, provided the original author(s) or licensor are credited and that the original publication in this journal is cited, in accordance with accepted academic practice. No use, distribution or reproduction is permitted which does not comply with these terms. 